\title{
Unsuspected Acquired Hemophilia in a Patient with Severe Postpartum Hemorrhage
}

\author{
${ }^{1}$ Shobhana Patted, ${ }^{2}$ BR Desai, ${ }^{2}$ Jayashree Ruge, ${ }^{2}$ Yeshita V Pujar, ${ }^{2}$ Bhavana Y Sherigar \\ ${ }^{1}$ Professor, Department of Obstetrics and Gynecology, KLE University's JN Medical College, Belgaum, Karnataka, India \\ ${ }^{2}$ KLE University's JN Medical College, Belgaum, Karnataka, India
}

\begin{abstract}
Correspondence: Shobhana Patted, Professor, Department of Obstetrics and Gynecology, KLE University's JN Medical College Belgaum, Karnataka, India, e-mail: klemrc.bgm@gmail.com
\end{abstract}

\section{ABSTRACT}

A 24-year-old healthy multigravid patient developed postpartum hemorrhage 3 days post normal delivery. She underwent curettage $\times 3$ followed by laparotomy and hysterectomy. Postoperatively she developed a subrectus hematoma that required laparotomy and drainage. Persistent bleeding per vaginum prompted a third laparotomy for internal iliac ligation, which could not be carried out due to massive retroperitoneal hematoma. She received multiple transfusions of blood and blood products. Her APTT was prolonged but DIC screen was negative. Prolonged APTT was not corrected by addition of normal plasma. This pointed to autoantibodies to factor VIII. She responded to immunosuppression with prednisolone and cyclophosphamide.

Keywords: Postpartum hemorrhage, Acquired hemophilia, Prolonged APTT, Retroperitoneal hematoma.

\section{INTRODUCTION}

Severe postpartum hemorrhage remains an important cause of maternal mortality. Most often it is due to uterine atony however other cause may exist. Acquired hemophilia is a rare disorder and the hemorrhage resulting from such a condition is difficult to treat. The diagnosis and treatment of this condition poses a serious challenge to an obstetrician. We report a case of acquired hemophilia managed successfully by us.

\section{CASE REPORT}

A 24-year-old para 3 was referred to us with postpartum hemorrhage. She had previous two normal deliveries. She apparently had an uncomplicated home delivery 18 days back. Three days after delivery, patient had brisk postpartum hemorrhage, for which she had undergone uterine curettage three times, in a span of 10 days. On admission, she was pale but stable hemodynamically, and had active bleeding vaginally. Ultrasonography did not reveal any retained placental fragments. In view of persistent bleeding, hysterectomy was performed. Postoperatively patient developed large hematoma under the dressing with continuous ooze from the incision site, which persisted in spite of pressure dressing ultrasonography of the abdomen confirmed the presence of hematoma under the rectus sheath measuring $7 \times 8 \mathrm{~cm}$. Laparotomy and evacuation of the clot was done and there was no other active source of internal bleeding seen. Investigation revealed hemoglobin of $8 \mathrm{gm} / \mathrm{dl}$,

Date of Received: 21-08-10

Date of Acceptance: 15-10-10

Date of Publication: Jan. 2011 white blood cell count and platelet count were normal. Prothrombin time was 12 seconds (control 13s) and activated partial thromboplastin time was 85 seconds (control 30s) with international normalized ratio (INR) of 1.04. Fibrinogen was within normal limit. No explanation for prolonged APTT was sought at this time.

On third postoperative day, patient developed a further episode of vaginal bleeding which was profuse with passage of clots. She was losing around $800 \mathrm{ml}$ of blood per day due to persistent continuous bleeding and the desperate clinical condition. Patient was taken for third hemostatic laparotomy and internal iliac ligation, however on laparotomy there was retroperitoneal hematoma and the whole anatomy was obscured. Abdomen was closed without any intervention leaving an intraabdominal and intrafacial drain. Throughout admission she was given fresh blood and frozen plasma, packed cells, and tranaxemic acid. Conservative approach was continued. Though patient was hemodynamically stable, she continued to bleed actively per vaginum. An intensivist opinion was sought and a presumptive diagnosis of DIC was made. Despite a hysterectomy, subsequent two laparotomies, constant support of fluids, blood and packed cells, the bleeding persisted. At this point her APTT was still noted to be prolonged 98 seconds (control 30s). This critical clinical features led us to do Medline search result using MeSH words prolonged APTT and PPH. Our search revealed acquired hemophilia as a rare cause of $\mathrm{PPH}$. Accordingly plasma correction test was done. It confirmed persistent prolongation of APTT, which was not corrected with addition of normal plasma. Fresh frozen plasma was sent to a referral laboratory for Bethesda assay, which was 24 Bethesda units. Test results of SLE, antinuclear antibody and anticardiolipin IgG were negative. 
Patient was commenced on immunosupression therapy with prednisolone $1 \mathrm{mg} / \mathrm{kg}$ and cyclophosphamide $100 \mathrm{mg}$ per day. Throughout her hospital stay, she was transfused 29 units of whole blood, 28 units of fresh frozen plasma and I unit of packed cell. Over the next 2 days, the response was evident by decreasing vaginal bleeding and normal APTT. She was continued with high dose steroids until all bleeding ceased. Her subsequent stay in the hospital was uneventful. Approximately 4 weeks later, her Bethesda assay was negative, and the dose of immunosuppressant drugs was tapered gradually. Patient made a good recovery.

\section{DISCUSSION}

Severe postpartum hemorrhage is one of the major cause of maternal deaths worldwide, ${ }^{1}$ and an estimated $95 \%$ of these deaths occur in sub-Saharan Africa and Asia. ${ }^{2}$ Postpartum hemorrhage accounts to approximately 25 to $30 \%$ of maternal deaths in India. ${ }^{3}$ Most often the cause is due to atonic uterus and is managed by medical or surgical interventions.

Acquired hemophilia is a rare disorder reported to occur in 0.2 to 1 per million population per year. ${ }^{4,5}$ It occurs as a result of the spontaneous development of auto antibodies against factor VIII. ${ }^{6}$ These patients can present with severe life threatening bleeding episodes. It is associated with autoimmune disorders (systemic lupus erythematosus, rheumatoid arthritis, asthma), neoplastic disorders, drug hypersensitivity, and pregnancy, however $50 \%$ of cases are idiopathic. ${ }^{6-8}$ Pregnancy or postpartum states are described as the associated factor in $7^{9}$ to $11 \%{ }^{6}$ of patients. It is most commonly seen 1 to 4 months after delivery, although cases appearing after 1 year have been described. $^{10}$

Patients with this disorder often present with dramatic hemorrhage into soft tissues and muscles, hematuria, hematemesis or prolonged postpartum or postoperative bleeding. ${ }^{11}$ Retroperitoneal bleeding can present particular diagnostic difficulties and is frequently fatal. ${ }^{5,12}$ Bleeding from any site may be prolonged and difficult to control. Death directly or indirectly attributable to acquired hemophilia has been reported in 10 to $22 \%$ of affected patients. ${ }^{5,9}$ The pattern of bleeding is different to that observed in congenital hemophilia, in particular spontaneous hemarthrosis is infrequent.

The potency of these inhibitor in pregnancy is rather low in majority of cases with median titer of about 20 Bethesda unit. ${ }^{13,14}$ Pregnancy associated acquired hemophilia is characterized in majority of the cases by a spontaneous disappearance of the inhibitor after a mean period of 30 months. ${ }^{10,15}$ Mortality rates also tends to be lower in this subset of patients ranging between 0 and $6 \% .^{13,14}$

As bleeding associated with factor VIII, inhibitor can be life threatening and responds poorly to traditional treatment of postpartum hemorrhage, and it is very important to diagnose this condition. The diagnosis of acquired hemophilia in a general hospital poses serious challenge to the managing obstetrician for several reasons. The condition may be undiagnosed or diagnosed late because of the rarity of this disorder, the non- availability specialty service of hematologist and diagnosis requires specific laboratory analysis for both factor VIII and inhibitor levels which needs sending the sample to a referral laboratory, which may take several hours to achieve and may not be easily available. In the absence of any history of bleeding tendency or conditions associated with coagulations disorder, and it is not usual to screen for coagulopathy in pregnancy.

However, there are certain significant clues for diagnosis, which include prolonged and unexplained postpartum bleeding in a woman with no history of bleeding disorder. Suspicious abnormal bleeding tendency should be investigated urgently with a clotting screen. The prolongation of activated partial thromboplastin time with normal prothrombin time is the hallmark of laboratory diagnosis. The bleeding time and platelet functions are normal. An increased APTT that cannot be corrected by the addition of normal plasma indicates a clotting inhibitor, and it needs to be further investigated by hematogist. ${ }^{16}$ Normal results in a clotting screen rule out this abnormality.

Management of acquired hemophilia is difficult, expensive, and normally carried out in specialist center. There are two strategies for the treatment of these patients; the treatment of active bleeding and immunosuppression to eliminate inhibitors. Options for the treatment of bleeding episodes are human or porcine factor VIII concentrate, activated prothrombin complex concentrates, and recombinant human activated factor VII. ${ }^{17}$ Immunosuppressive drugs that are used to eradicate the antibodies are prednisolone, cyclophospamide, azathioprine and cyclosporin. Treatment with intravenous immunoglobulins has been successful, and the anti-CD20 antibody Rituximab has also been found effective. ${ }^{18,19}$

An important clinical issue regarding pregnancy related inhibitors is its possible recurrence in the subsequent pregnancies, which makes counseling of women with this profile essential. In this regard, results are conflicting and although most series have found that inhibitors tend to not to recur in patients who attain a complete remission ${ }^{10,20}$

This case illustrates the importance of noting and acting upon abnormalities of baseline blood tests. The APTT was abnormal early in her admission but the importance of the abnormal ratio was not appreciated until the Medline search, which revealed the possibility of this rare disorder.

In conclusion, acquired hemophilia can manifest as severe postpartum hemorrhage. An isolated elevated APTT with normal prothrombin time in an other wise asymtomatic patient should not be ignored, requires investigation and immediate refferal, however these options are not widely available in all hospitals and the financial constraint may prevent optimal management in these patients. 


\section{REFERENCES}

1. Gilbert L, Porter W, Brown VA. Postpartum haemorrhage: A continuing problem. Br J Obstet Gynaecol 1987;94:67-71.

2. World Health Organizations. Managing complications in pregnancy and childbirth. Maternal mortality in 2000: Estimates developed by WHO, UNICEF and UNFPA. Geneva: World Health Organization, Department of Reproductive Health and Research (RHR) 2003.

3. Annual report 2003-2004. Ministry of Health and Family Welfare, Government of India, New Delhi.

4. Duran-Suarez JR. Incidence of circulating anticoagulants in normal population. Acta haematol 1982;67:217-19.

5. Lottenberg R, Kentro TB, Kitchens CS. Acquired haemophilia: A natural history study of 16 patients with factor VIII inhibitors receiving little or no therapy. Arch Intern Med 1987;147:107781.

6. Kessler CM, Ludlam CA. The treatment of acquired factor VIII inhibitors: Worldwide experience with porcine factor VIII concentrate. Semin Hematol 1993;30:22-27 (Suppl 1).

7. Ludlam CA, Morrison AE, Kessler C. Treatment of acquired Hemophilia. Semin Hematol 1994; 31:16-19 (Suppl 4).

8. Morrison AE, Ludlam CA. Acquired haemophilia and its treatment. Br J Haematol 1995; 89:231-236.

9. Green D, Lechner K. A survey of 215 non-hemophilic patients with inhibitors to factor VIII. Thromb Haemost 1981;45:20003.

10. Michiels JJ. Acquired hemophilia A in women Postpartum: Clinical manifestations, diagnosis, and treatment. Clinical and applied thrombosis/hemostasis 2000;6:82-86.

11. Morrison AE, Ludlam CA, Kessler C. Use of porcine factor VIII in the treatment of patients with acquired hemophilia. Blood 1993;81:1513-20.
12. Roy V, Tillyer ML, Colvin BT. Acute abdominal pain due to an acquired disorder of coagulation. British Medical Journal 1998;296:1460-61.

13. Hauser I, Schneider B, Lechner K. Post-partum factor VIII inhibitors: A review of the literature with special reference to the value of steroid and immunosuppressive treatment. Thrombosis and Haemostasis 1995;73:1-5.

14. Silymoss S. Postpartum acquired factor VIII inhibitors: Results of a survey. American Journal of Haematology 1998;59:1-4.

15. Michiels JJ, Hamulyak K, Nieuwenhuis HK, Novakova I, Van Vliet HH. Acquired haemophilia A in women post-partum: Management of bleeding episodes and natural history of the factor VIII inhibitor. European Journal of Haematology 1997;59:105-18.

16. Kadir RA, Koh MB, Lee CA, Pasi KJ. Acquired haemophilia, An unusual cause of severe postpartum haemorrhage. British Journal of Obstetrics and Gynaecology 1997;104:854-56.

17. Arkin S, Blei F, Fetten J. Human coagulation factor FVIIa (recombinant) in the management of limb-threatening bleeds unresponsive to alternative therapies: Results form the NovoSeven emergency-use programme in patients with severe haemophilia or with acquired inhibitors. Blood Coagul Fibrinolysis 2000;11:255-59.

18. Kain S, Copeland TS, Leahy MF. Treatment of refractory autoimmune (acquired) haemophilia with anti-CD20 (rituximb). Br J Haematol 2002;119:578.

19. Wiestner A, Cho HJ, Asch AS. Rituximab in the treatment of acquired factor VIII inhibitors. Blood 2002;100:3426-28.

20. Coller BS, Hultin MB, Hoyer LW, Miller F, Dobbs JV, Dosik $\mathrm{MH}$, Berger ER. Normal pregnancy in a patient with a prior postpartum factor VIII inhibitor, with observations on pathogenesis and prognosis. Blood 1981;58:619-24. 


\section{Book Review}

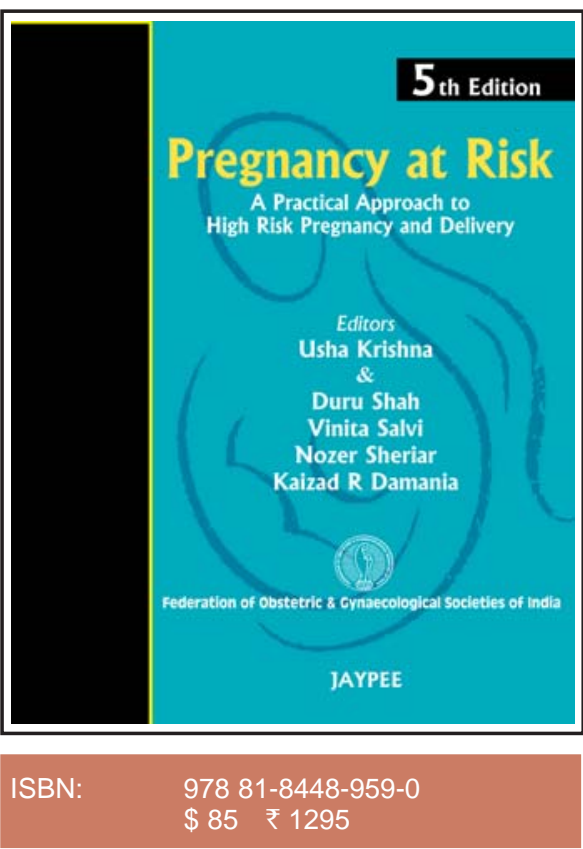

Publishers: Jaypee Brothers Medical Publishers (P) Ltd.

Also Available online at www.jaypeebrothers.com

FOGSI Publication
Usha Krishna

MD DGO FICS FICOG

Past President, FOGSI, MOGS, Ex-Hon. Prof. KEM Hospital and

GS Medical College

Consultant Obstetrician and Gynecologist, Bhatia General Hospital

Director, Clinic for Women, Laburnum Road

Gamdevi, Mumbai, Maharashtra, India

Duru Shah

MD FRCOG FCPS FICS FICOG FICMCH DGO DFP

Chairman: Gynaecworld

Professor and Consultant Obstetrician and Gynecologist

Breach Candy Hospital and Research Center

Jaslok Hospital and Research Center

Sir Hurkisondas Nurrotumdas Hospital and Research Center Mumbai, Maharashtra, India

Vinita Salvi

MD DNBE FCPS DGO DFP

Consultant Obstetrician and Gynecologist

SevenHills Hospital, Mumbai

Ex-Professor of Obstetrics and Gynecology and

Officer in-Charge, ICMR Regional Center for Research in

Human Reproduction, Seth GS Medical College and

KEM Hospital, Mumbai, Maharashtra, India

Nozer Sheriar

MD DNBE FCPS DGO

Consultant Obstetrician and Gynecologist

Breach Candy, Holy Family, Masina and Parsee General Hospitals Gynecological Endoscopist, Bhatia and BARC Hospitals

Deputy Secretary General: FOGSI

Mumbai, Maharashtra, India

Kaizad R Damania

MD DNBE FCPS DGO DFP

Professor of Obstetrics and Gynecology Seth GS Medical College and Nowrosjee Wadia Maternity Hospital, Mumbai, Maharashtra, India

\section{Pregnancy at Risk \\ A Practical Approach to High Risk Pregnancy and Delivery}

This is a textbook for clinicians practicing obstetrics and postgraduate students. It comprehensively covers the "at risk" situations in each trimester and its evidence-based management. It has over 100 chapters contributed by over 150 authors, each an expert in their field. Also it comprehensively covers medical disorders and obstetric complications in pregnancy, infections during pregnancy, and effects of drugs and toxins in pregnancy. It deals with labor and delivery in pregnancy at risk as well as invasive procedures during pregnancy, like fetal reduction, chorionic villus sampling, amniocentesis as well as ablation of placental vessels in twin to twin transfusion.
Mala Arora

FRCOG, FICOG, FICMCH, DA (UK), D'Obst (Ire) Vice President FOGSI
It has chapters on medical therapy to the fetus and intrauterine fetal surgery for diaphragmatic hernia, posterior urethral valves and placement of shunts for hydrocephalus as well as hydrothorax. It has chapters on gestational trophoblastic disease and laparoscopy in pregnancy.

It is a comprehensive textbook of obstetrics that will be an asset to the library and a good reference book for the practicing clinicians. The first edition of the book was published in 1993 and it has seen regular updates since then. It is hardbound with color illustrations and is also priced economically. 